\title{
Shen Song Yang Xin Capsule Combined with Antiarrhythmic Drugs, a New Integrative Medicine Therapy, for the Treatment of Frequent Premature Ventricular Contractions (FPVC): A Meta-Analysis of Randomized Controlled Trials
}

\author{
Jie Wang, Jun Li, and Bo Feng \\ Department of Cardiology, Guang'anmen Hospital, China Academy of Chinese Medical Sciences, \\ Beixiange No. 5, Xicheng District, Beijing 100053, China \\ Correspondence should be addressed to Bo Feng; fengbo263@163.com
}

Received 3 December 2013; Revised 13 January 2014; Accepted 27 January 2014; Published 18 March 2014

Academic Editor: Xingjiang Xiong

Copyright (C) 2014 Jie Wang et al. This is an open access article distributed under the Creative Commons Attribution License, which permits unrestricted use, distribution, and reproduction in any medium, provided the original work is properly cited.

\begin{abstract}
Objective. To evaluate the beneficial and adverse effects of Shen Song Yang Xin Capsule (SSYX Capsule) combined with antiarrhythmic drugs for the treatment of frequent premature ventricular contractions (FPVC). Methods. Seven electronic databases were searched to retrieve any potential randomized controlled trials (RCTs) designed to evaluate the clinical efficacy of SSYX Capsule combined with Antiarrhythmic Drugs for FPVC reported in any language, with total effect for FPVC and number of ventricular premature contraction as the main outcome measure. The methodological quality of the included studies was assessed using criteria from the Cochrane Handbook for Systematic Review of Interventions, Version 5.1.0, and analysed using RevMan 5.1.0 software. Results. Sixteen RCTs of SSYX Capsule were included. The methodological quality of the trials was generally evaluated as low. The results of meta-analysis showed that SSYX Capsule combined with antiarrhythmic drugs was more effective in total effect for FPVC and number of ventricular premature contraction compared with Antiarrhythmic Drugs in patients with FPVC or FPVC complicated by other diseases. Ten of the trials reported adverse events, indicating that the safety of SSYX Capsule is still uncertain. Conclusions. There is some but weak evidence about SSYX Capsule combined with antiarrhythmic drugs appearing to be more effective in total effect for FPVC and number of ventricular premature contraction in patients with FPVC and its complications.
\end{abstract}

\section{Introduction}

Frequent premature ventricular complexes (PVCs) are a common occurrence in clinical practice. It is responsible for considerable morbidity and mortality. It may cause haemodynamic deterioration and reversible left ventricular (LV) dysfunction and can act as markers to other cardiac diseases such as cardiomyopathy and ischemic heart disease [1]. Recent research demonstrated that the quantitative additional mortality risk on exercise test (ET) presented by frequent PVCs is similar to ischemia [2]. Clinical presentation of PVCs may be quite variable, ranging from an incidental finding on electrocardiogram (ECG) to congestive heart failure (CHF) [3]. For patients with symptoms that can be attributed to PVCs, the medical therapy of beta blockers or class I or III antiarrhythmic agents is effective and recommended. However, some patients cannot tolerate medical therapy. Currently, radiofrequency catheter ablation (RFCA) been increasingly used of symptomatic PVCs has been reported to be a safe and efective treatment option $[4,5]$.

With increasing popularity of complementary and alternative medicine among patients with PVCs, TCM (Traditional Chinese Medicine) is becoming more and more frequently used both in China and western countries [6]. Shen Song Yang Xin Capsule (SSYX Capsule) is a widely used Chinese medicine with antiarrhythmic effect. Many clinical researches showed that it can effectively improve symptoms of heart palpitations, chest tightness, shortness of breath, insomnia, fatigue, and so on [7-9]. Mechanism of antiarrhythmic effect is mainly due to blockade effect 
of different ion channels $[10,11]$. Although there has been much research on SSYX Capsule combined with Western medicine for the treatment of FPVC, there is no critically appraised evidence such as systematic reviews or metaanalyses on potential benefits. This report aims to evaluate the beneficial and adverse effects of SSYX Capsule combined with antiarrhythmic drugs for the treatment of FPVC on total effect for FPVC and number of ventricular premature contraction.

\section{Materials and Methods}

2.1. Database and Search Strategies. We selected all the clinical trials about SSYX Capsule combined with antiarrhythmic drugs for treatment of FPVC in the Chinese National Knowledge Infrastructure (CNKI), the Chinese Biomedical Literature Database (CBM), the Chinese Scientific Journal Database (VIP), PubMed, and the Cochrane Central Register of Controlled Trials in the Cochrane Library (May 2013). We also searched the reference list of retrieved papers. Databases in Chinese were searched to retrieve the maximum possible number of trials of SSYX Capsule for FPVC because it is mainly used in China. The following search terms were used individually or combined: "frequent premature ventricular contractions," "frequent premature ventricular beats," "Shen Song Yang Xin Capsule," "combined with," "controlled clinical trial," "clinical trial," and "Randomized Controlled Trials.”

2.2. Inclusion and Exclusion Criteria. All randomized controlled trials (RCTs) of patients with FPVC that studied prescriptions based on SSYX Capsule combined with antiarrhythmic drugs compared with antiarrhythmic drugs were included. There were no restrictions on language, population characteristics, and publication type. The primary outcome measure was total effect for FPVC and number of ventricular premature contractions. Duplicated publications reporting the same groups of participants were excluded.

2.3. Data Extraction and Quality Assessment. Two authors (Jun Li and Bo Feng) conducted the literature searching, study selection, and data extraction independently. The extracted data included the title of the study, authors, year of publication, article source, study size, total number of cases (Treatment/Control), diagnosis standard, details of methodological information, and treatment course, and clinical standards as well as the details of the control interventions, outcomes, and adverse effects for each study. Disagreement was resolved by discussion and reached consensus through a third party (Jie Wang). The methodological quality of included trials was assessed according to the Cochrane Handbook for Systematic Review of Interventions, Version 5.1.0 [12]. The assessment used the following 6 criteria, including random sequence generation (selection bias), allocation concealment (selection bias), blinding of participants and personnel (performance bias), blinding of outcome assessment (detection bias), incomplete outcome data (attrition bias), selective reporting (reporting bias), and other bias.
2.4. Data Synthesis. RevMan 5.1.0 software provided by the Cochrane Collaboration was used for data analyses. Dichotomous data were expressed as relative risk (RR) and continuous outcomes were presented as weighted mean difference (WMD), while 95\% confidence intervals (CI) were calculated for both. Meta-analysis was performed if the intervention, control, and outcomes were the same or similar. The statistical heterogeneity was presented as significant when the $I$ square $\left(I^{2}\right)$ value exceeded $50 \%$ or $P<0.1$. In the absence of significant heterogeneity, we pooled data using fixed effects model $\left(I^{2}<50 \%\right)$; otherwise we used random effects model $\left(I^{2}>50 \%\right)$.

\section{Results}

3.1. Description of the Included Trials. After the primary search of the five databases, 534 potentially relevant trials were screened out from electronic and manual searches. The majority were excluded because duplicates were removed and some included irrelevant titles and abstracts. Only 127 studies were retrieved. Following review of the titles and abstracts, several studies were excluded, and only 46 studies remained. 21 trials did not use the criteria of total effect for FPVC and number of ventricular premature contractions as the outcome measure. 4 trials have no control group. 3 clinical trials included Chinese herbal formula as the control group and 2 trials had no data for extraction. In the end, sixteen RCTs were to be reviewed [13-28]. The study selection process is summarised in a flow chart (Figure 1). All of the trials were conducted in China and published in Chinese. The characteristics of the sixteen RCTs are summarised in Table 1.

The sixteen RCTs involved a total number of 1115 patients with FPVC. One trial [15] study FPVC with sick sinus syndrome, four trials $[13,20,26,28]$ study elderly FPVC and other studies FPVC without detailed information. Of those, one [15] used an international consensus on nomenclature and classification of FPVC developed by ACC/AHA/ESC 2008 Guidelines for the Management of Patients with FPVC (ACC/AHA/HRS 2008). Two trials demonstrated that patients with FPVC diagnosis were more than 720/24 h [13, 16]. Four trials of FPVC diagnosis were more than $30 / \mathrm{h}[17,19$, $24,26]$. One trial used diagnosis criteria of more than $5 / \mathrm{min}$ [18]. One trial used diagnosis criteria of more than 1000/24 h. One trial used diagnosis criteria of more than $7200 / 24 \mathrm{~h}$. The rest of the trials $[14,21,22,25,27,28]$ only demonstrated patients with FPVC diagnosis by electrocardiogram and 24hour Holter without detailed information.

The interventions of all sixteen trials [13-28] included SSYX Capsule combined with antiarrhythmic drugs. One control used conventional therapy with antiarrhythmic drugs with no detailed information [15]. The other controls were antiarrhythmic drugs alone. The total treatment duration ranged from 1 month to 6 months. Thirteen trials $[13,16,19$, 21] specified clinical standards of FPVC by "Clinical research guideline of medicine for cardiovascular system by Ministry of Public Health (1998)." The clinical standards of FPVC by "Clinical research guideline of medicine for cardiovascular system by Ministry of Public Health (1998)" were described 


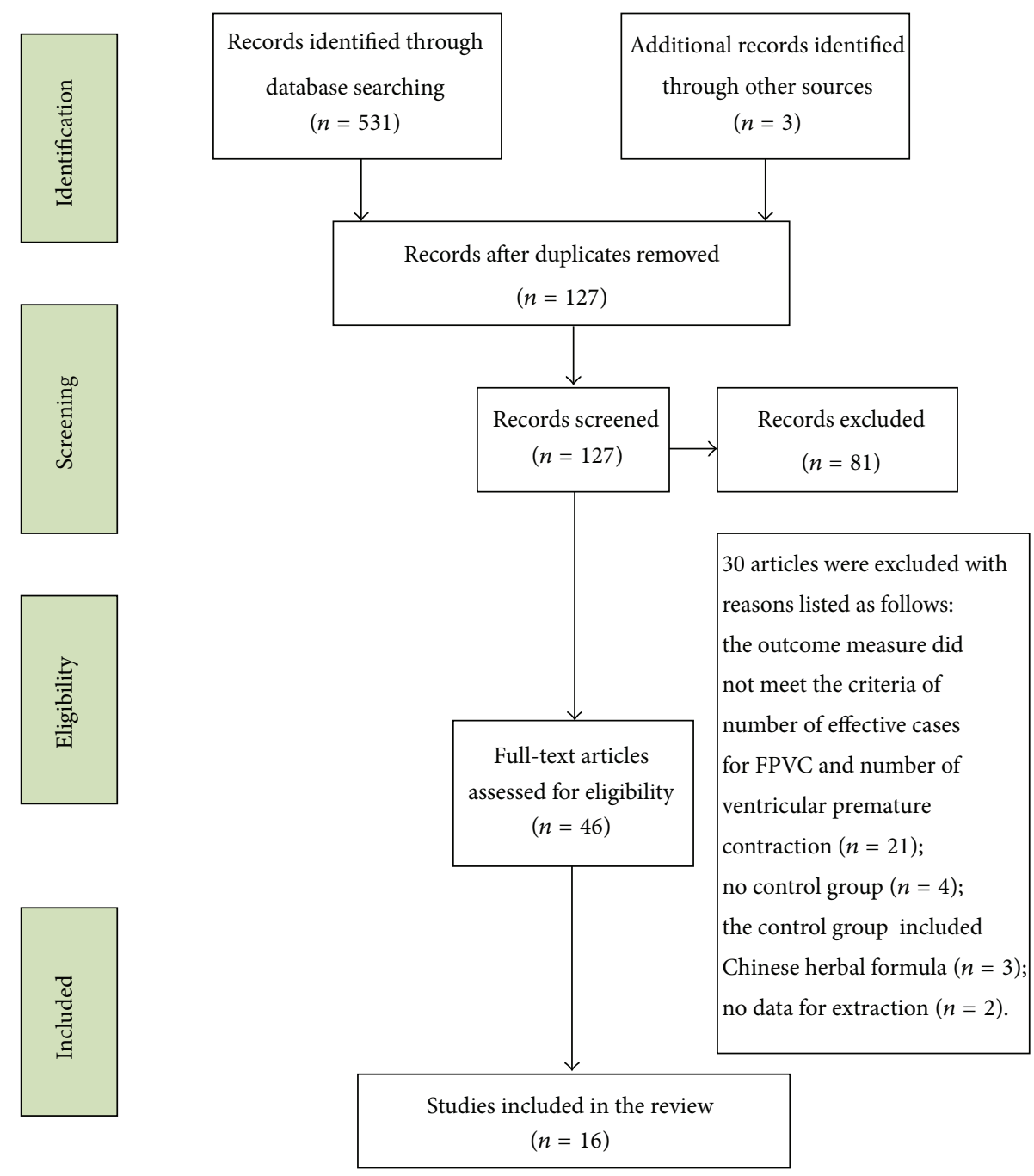

FIGURE 1: Study selection process.

as follows. Marked effective result: ventricular premature contraction diminished or decreased more than $90 \%$ in 24-hour Holter. Chest distress and palpitation symptoms disappeared or improved. Effective result: ventricular premature contraction diminished or decreased more than $50 \%$ in 24-hour Holter. Chest distress and palpitation symptoms partially disappeared or improved. Ineffective result: ventricular premature contraction diminished or decreased less than $50 \%$ in 24-hour Holter. Chest distress and palpitation symptoms had no improvement or aggravated.

All the sixteen trials [13-28] used the total effect for FPVC as the main outcome measure, and five of the sixteen trials $[15,17,19,20,24]$ used the number of ventricular premature contraction as the main outcome measure. Ten of the included trials [13-15, 17, 20, 22-24, 26, 27] described adverse effects in detail.

3.2. Methodological Quality of the Included Trials. The majority of the included trials were assessed to be of general poor methodological quality according to the predefined quality assessment criteria (As shown in Table 2). The randomized allocation of participants was mentioned in all trials. However, only one trial stated the methods for sequence generation by random number table [16]. None of the trials calculated an estimation of the pretrial sample size; there may be insufficient power to ensure appropriate estimation of the therapeutic effect. Allocation concealment and blinding of outcome assessment were not mentioned in all trials.

\subsection{Effects of the Interventions}

3.3.1. Effective Frequency Number for FPVC. All the sixteen trials [13-28] used the total effect for FPVC as an outcome measure. These sixteen trials compared the combination of SSYX Capsule plus antiarrhythmic drugs with antiarrhythmic drugs alone. Trial results for the sixteen independent trials were homogeneous, $\chi^{2}=7.43, \mathrm{df}=15,(P=$ $0.94) ; I^{2}=0 \%$, requiring the use of the fixed effects model for statistical analysis. The effective frequency number after SSYX Capsule combined with antiarrhythmic drugs 


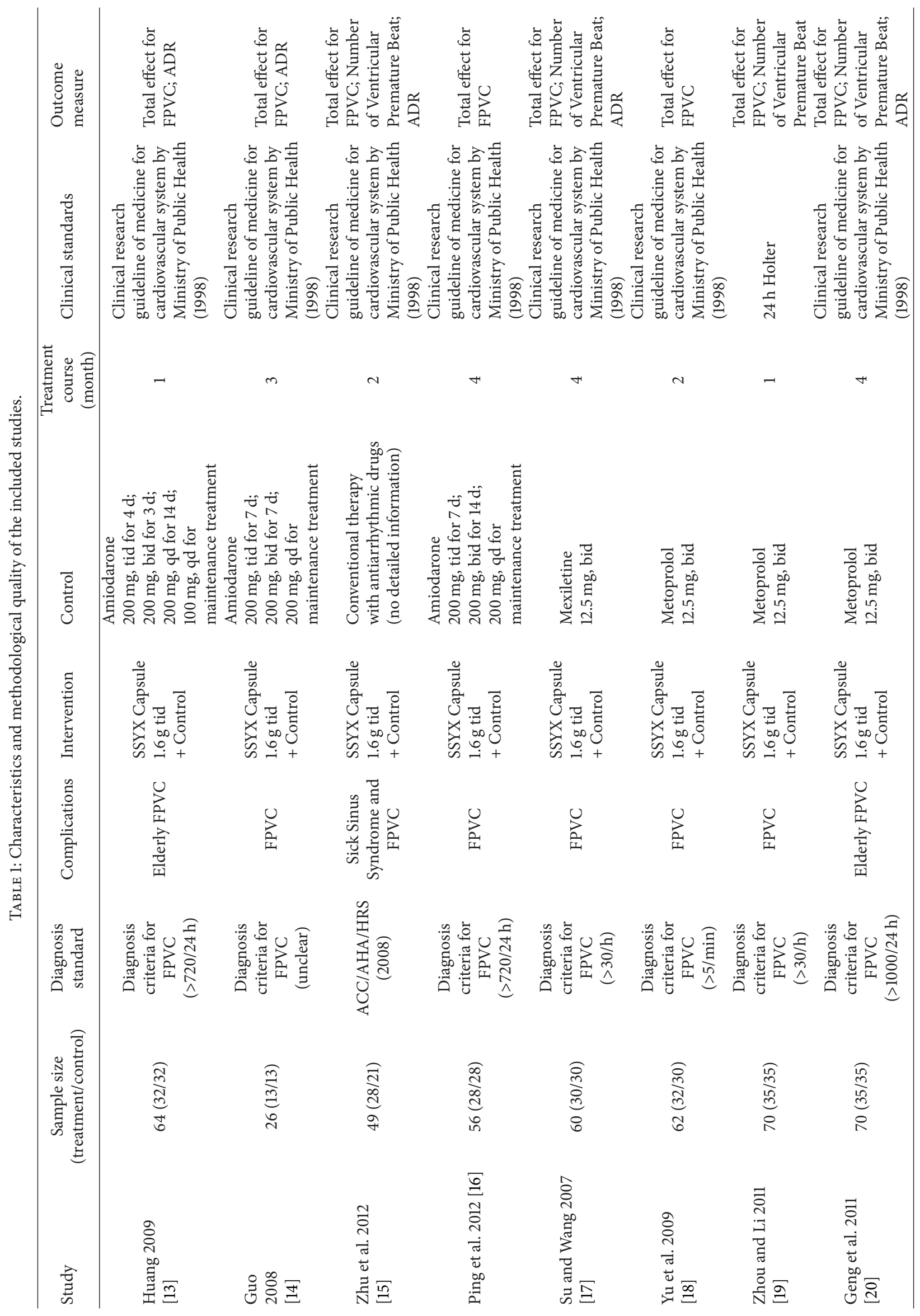




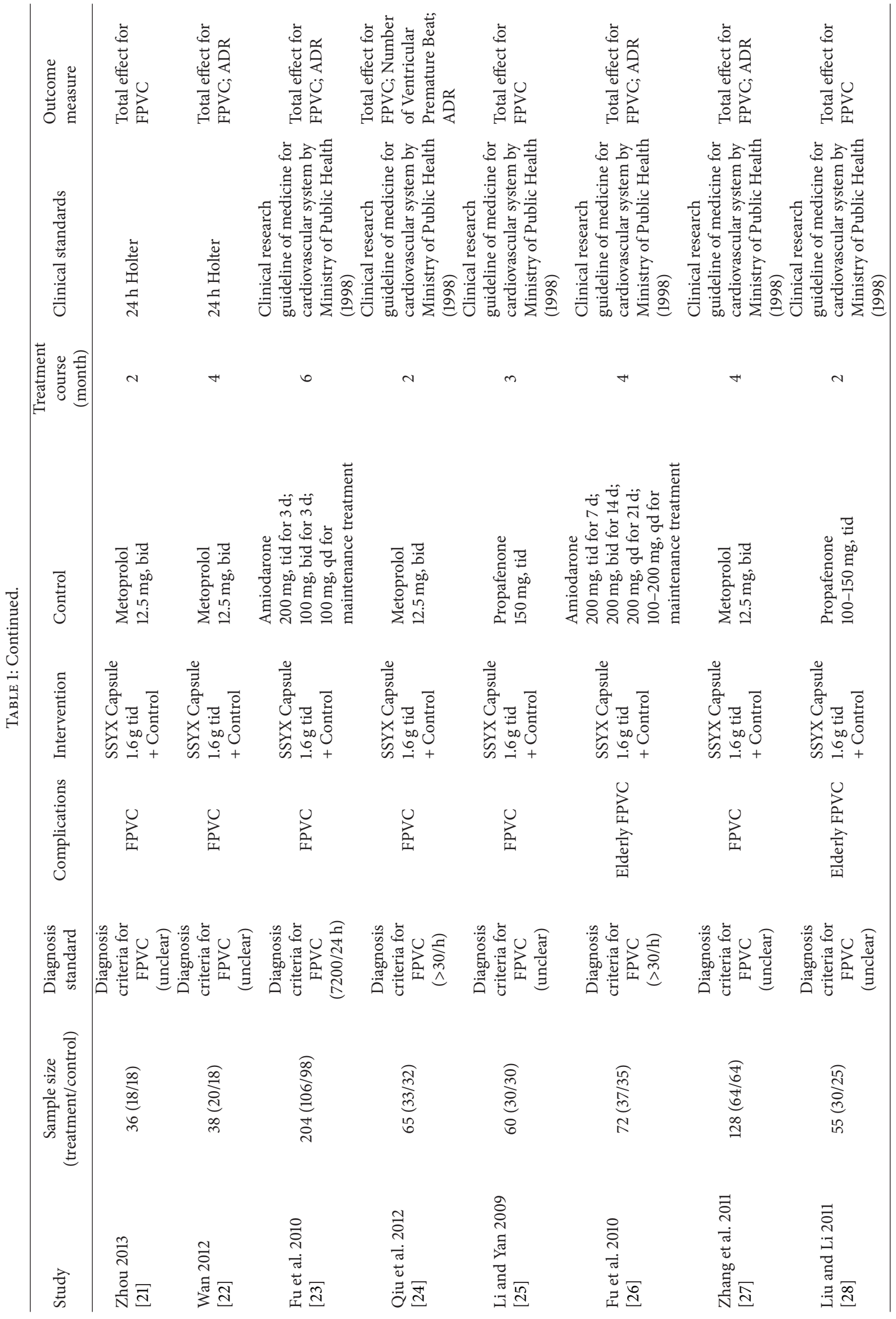


TABLE 2: Quality assessment of included randomized controlled trials.

\begin{tabular}{|c|c|c|c|c|c|c|c|}
\hline Included trials & $\begin{array}{l}\text { Random } \\
\text { sequence } \\
\text { generation }\end{array}$ & $\begin{array}{c}\text { Allocation } \\
\text { concealment }\end{array}$ & $\begin{array}{c}\text { Blinding of } \\
\text { participants and } \\
\text { personnel }\end{array}$ & $\begin{array}{l}\text { Blinding of } \\
\text { outcome } \\
\text { assessment }\end{array}$ & $\begin{array}{l}\text { Incomplete } \\
\text { outcome data }\end{array}$ & $\begin{array}{l}\text { Selective } \\
\text { reporting }\end{array}$ & $\begin{array}{c}\text { Other sources } \\
\text { of bias }\end{array}$ \\
\hline $\begin{array}{l}\text { Huang } 2009 \\
{[13]}\end{array}$ & Unclear & Unclear & Unclear & Unclear & No & No & Unclear \\
\hline $\begin{array}{l}\text { Guo } \\
2008 \\
{[14]}\end{array}$ & Unclear & Unclear & Unclear & Unclear & No & No & Unclear \\
\hline $\begin{array}{l}\text { Zhu et al. } 2012 \\
\text { [15] }\end{array}$ & Unclear & Unclear & Unclear & Unclear & No & No & Unclear \\
\hline $\begin{array}{l}\text { Ping et al. } 2012 \\
{[16]}\end{array}$ & $\begin{array}{l}\text { Table of } \\
\text { random } \\
\text { number }\end{array}$ & Unclear & Unclear & Unclear & No & No & Unclear \\
\hline $\begin{array}{l}\text { Su and Wang } \\
2007 \\
{[17]}\end{array}$ & Unclear & Unclear & Unclear & Unclear & No & No & Unclear \\
\hline $\begin{array}{l}\text { Yu et al. } \\
2009 \\
{[18]}\end{array}$ & Unclear & Unclear & Unclear & Unclear & No & No & Unclear \\
\hline $\begin{array}{l}\text { Zhou and Li } 2011 \\
{[19]}\end{array}$ & Unclear & Unclear & Unclear & Unclear & No & No & Unclear \\
\hline $\begin{array}{l}\text { Geng et al. } 2011 \\
{[20]}\end{array}$ & Unclear & Unclear & Unclear & Unclear & No & No & Unclear \\
\hline $\begin{array}{l}\text { Zhou } 2013 \\
{[21]}\end{array}$ & Unclear & Unclear & Unclear & Unclear & No & No & Unclear \\
\hline $\begin{array}{l}\text { Wan } 2012 \\
{[22]}\end{array}$ & Unclear & Unclear & Unclear & Unclear & No & No & Unclear \\
\hline $\begin{array}{l}\text { Fu et al. } \\
2010 \\
{[23]}\end{array}$ & Unclear & Unclear & Unclear & Unclear & No & No & Unclear \\
\hline $\begin{array}{l}\text { Qiu et al. } 2012 \\
{[24]}\end{array}$ & Unclear & Unclear & Unclear & Unclear & No & No & Unclear \\
\hline $\begin{array}{l}\text { Li and Yan } \\
2009 \\
{[25]}\end{array}$ & Unclear & Unclear & Unclear & Unclear & No & No & Unclear \\
\hline $\begin{array}{l}\text { Fu et al. } \\
2010 \\
{[26]}\end{array}$ & Unclear & Unclear & Unclear & Unclear & No & No & Unclear \\
\hline $\begin{array}{l}\text { Zhang et al. } 2011 \\
\text { [27] }\end{array}$ & Unclear & Unclear & Unclear & Unclear & No & No & Unclear \\
\hline $\begin{array}{l}\text { Liu and Li } 2011 \\
\text { [28] }\end{array}$ & Unclear & Unclear & Unclear & Unclear & No & No & Unclear \\
\hline
\end{tabular}

was more than antiarrhythmic drug treatment. The metaanalysis demonstrated a significant difference between the two groups. (MD: 3.21 [2.27, 4.54]; $P<0.00001$ ) (Figure 2).

3.3.2. Number of Ventricular Premature Contractions. Five trials $[15,17,19,20,24]$ used the number of ventricular premature contractions as an outcome measure. Trial results for the seven independent trials were not homogeneous, $\chi^{2}=$ 957.93, $\mathrm{df}=4,(P<0.00001) ; I^{2}=100 \%$, requiring the use of the random effects model for statistical analysis. The number of ventricular premature contractions in the SSYX Capsule combined with antiarrhythmic drug group was less than that of antiarrhythmic drug group, but the meta-analysis did not show significant beneficial effect in the combination group compared with the antiarrhythmic drug group ( $\mathrm{RR}-8.58$; 95\% CI $[-18.84,1.68] ; P=0.10$ ) (Figure 3).

3.4. Subgroup Analysis and Publication Bias. The number of trials was too small to conduct analysis of subgroup and publication bias.

3.5. Adverse Effects. Ten out of the included trials [13-15, $17,20,22-24,26,27]$ described adverse effects in detail. All the ten cases mentioned specific symptoms, including sleepiness, nausea, loss of appetite, stomach discomfort, and sinus bradycardia, in the SSYX Capsule combined with 


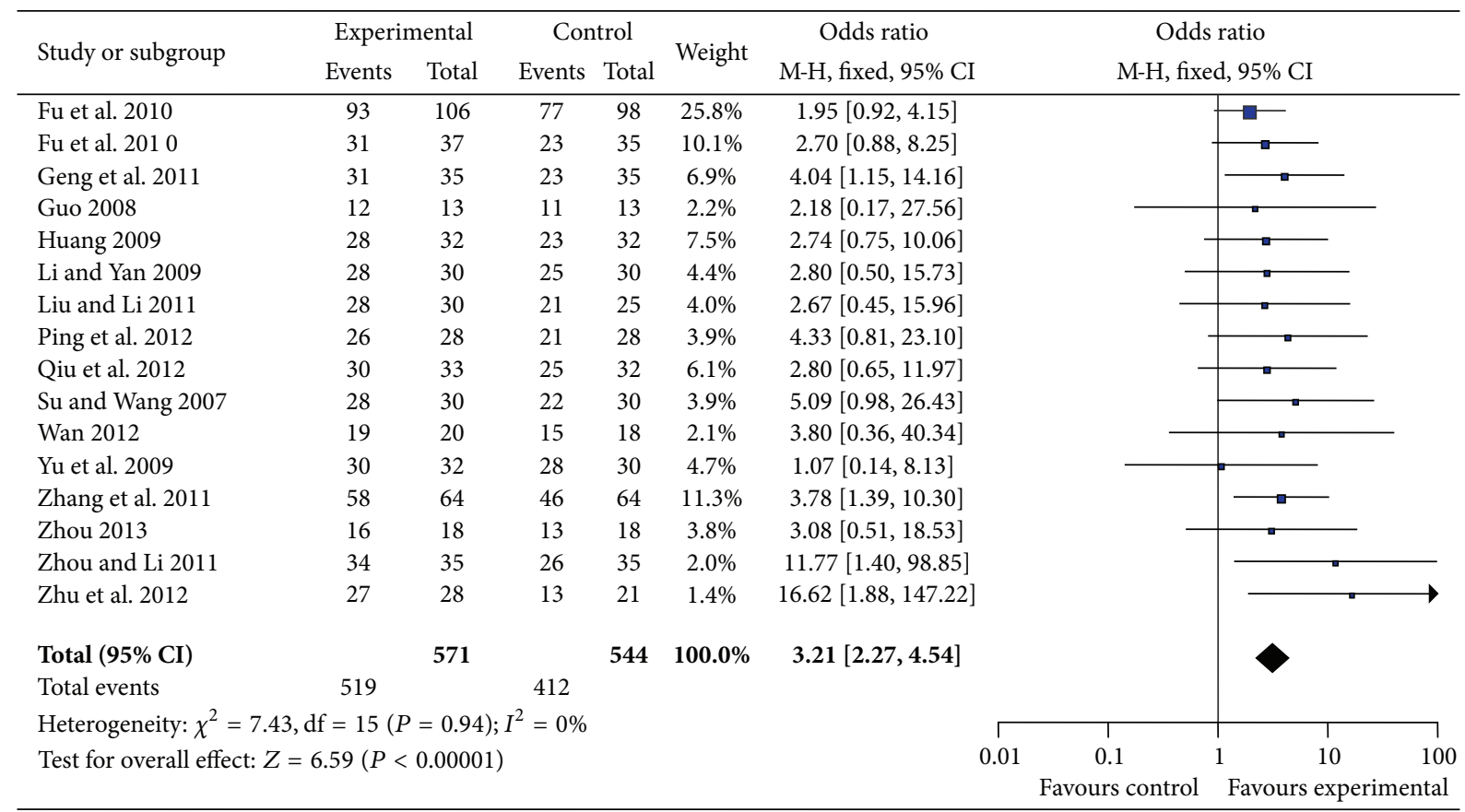

FIGURE 2: Analysis of total effect for FPVC. Forest plot of comparison: SSYX Capsule combined with antiarrhythmic drug group versus antiarrhythmic drug group.

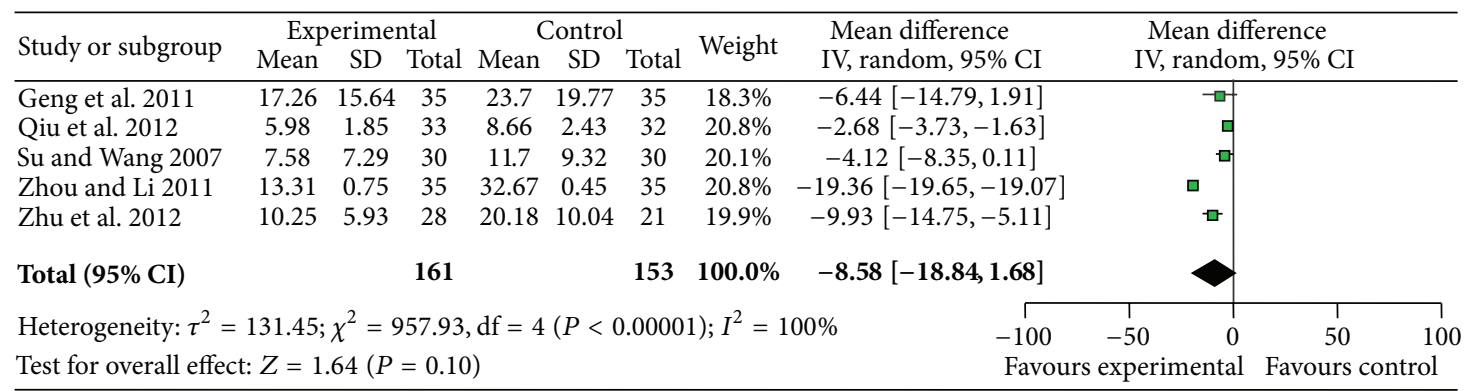

FIGURE 3: Analysis of number of ventricular premature contractions. Forest plot of comparison: SSYX Capsule combined with antiarrhythmic drug group versus antiarrhythmic drug group.

antiarrhythmic drugs group and I atrioventricular block, II atrioventricular block, sinus bradycardia, abdominal distension, and so on in the antiarrhythmic drugs group. These side effects may be related to the adverse effect of amiodarone. Eight trials $[13,14,17,20,22-24,26]$ mentioned adverse effects in both groups and mentioned specific symptoms. In the experimental group, one case of sleepiness was reported, seven cases of nausea, one case of loss of appetite, twenty cases of stomach discomfort, and seven cases of dizziness and one case demonstrated adverse effect of sinus bradycardia. In the controlled group, one case reported adverse effect of I atrioventricular block, one case I atrioventricular block and $U$ wave, thirteen cases nausea, abdominal distension, and stomach discomfort, ten cases sinus bradycardia, of which three cases were serious, two cases II atrioventricular block, two cases low blood pressure, four cases liver function damage, seven cases dizziness, and three cases Q-T interval prolongation and one case showed adverse effect of Q-T interval prolongation combined with paroxysmal ventricular tachycardia. One trial [15] reported adverse effects in the SSYX Capsule combined with antiarrhythmic drugs group of mildly stomach discomfort in four cases. One trial [27] reported adverse effects in the antiarrhythmic drugs group of sinus bradycardia in three cases. In general, the incidence of adverse reactions was lower in the treatment group compared with the control group.

\section{Discussion}

PVCs is common clinical heart disease. It can present with a wide spectrum of symptoms in patients with and without structural heart disease. In patients with structural heart disease, they may cause or aggravate the disease process, or be a consequence of the disease process itself. The main symptom of this disease in clinic is palpitation, which can 
induce feeling of heart pounding in patients or the feeling of dizziness and anxiety and even syncope. This disease not only cause abnormal work and life in patients, and even endanger the safety of life seriously.

Conventional medicine therapy can inhibit the deterioration of clinical symptoms of the patients in a short period of time but cannot reduce the physical and psychological suffering of patients fundamentally. TCM has long history and abundant experiences in treatment of clinical manifestations frequently reported by patients with frequent premature ventricular contractions. Currently, with wide acceptance of integrative medicine (IM) for primary health care worldwide, more and more people with CVDs select CAM/TCM to maintain their health for more efficacy and less adverse effect $[29,30]$. Although there are still some problems in this domain, evidence based IM is warranted in further researches.

This paper included 16 randomized trials and a total of 1115 participants. Shen Song Yang Xin Capsule combined with antiarrhythmic drugs, a new integrative medicine therapy, showed significant benefit on outcome of total effect and improving symptoms and signs as compared with conventional treatment for FPVC. However, due to the lowquality methodology and potential publication bias, a definite conclusion of the beneficial effectiveness of SSYX Capsule combined with antiarrhythmic drugs in treating FPVC could not be drawn. In addition, Shen Song Yang Xin Capsule is a traditional Chinese medicine. It should therefore be used under the guidance of TCM theory of "syndrome differentiation and treatment" [31-33]. Also, more guidance on the theory of traditional Chinese medicine is needed in the future.

\section{Conclusion}

There is encouraging evidence of the effect of SSYX Capsule combined with antiarrhythmic drugs on total effect for FPVC and number of ventricular premature contractions with FPVC. Due to the poor quality of experimental design and methodology, the evidence remains weak. More rigorous RCTs with strong design and high methodological quality will be needed to present a high level of evidence for the effectiveness of SSYX Capsule in treating FPVC.

\section{Conflict of Interests}

All authors manifest that there is no conflict of interests.

\section{Authors' Contribution}

Jie Wang, Jun Li, and Bo Feng contributed equally to this paper.

\section{Acknowledgment}

The current work was partially supported by the National Basic Research Program of China (973 Program, no.
2003CB517103) and the National Natural Science Foundation Project of China (no. 90209011).

\section{References}

[1] D. N. Duddleston, "Frequent premature ventricular contractions," Journal of Insurance Medicine, vol. 32, no. 4, pp. 262-265, 2000.

[2] J. R. Iacovino, "Comparison of the mortality between ischemia and frequent premature ventricular contractions on an exercise test," Journal of Insurance Medicine, vol. 33, no. 3, pp. 222-226, 2001.

[3] J. C. Adams, K. Srivathsan, and W. K. Shen, "Advances in management of premature ventricular contractions," Journal of Interventional Cardiac Electrophysiology, vol. 35, no. 2, pp. 137149, 2012.

[4] M. Takemoto, H. Yoshimura, Y. Ohba et al., "Radiofrequency catheter ablation of premature ventricular complexes from right ventricular outflow tract improves left ventricular dilation and clinical status in patients without structural heart disease," Journal of the American College of Cardiology, vol. 45, no. 8, pp. 1259-1265, 2005.

[5] C.-X. Huang, J.-J. Liang, B. Yang et al., "Quality of life and cost for patients with premature ventricular contractions by radiofrequency catheter ablation," Pacing and Clinical Electrophysiology, vol. 29, no. 4, pp. 343-350, 2006.

[6] J. Wang, X. J. Xiong, and B. Feng, "Cardiovascular effects of salvianolic acid B," Evidence-Based Complementary and Alternative Medicine, vol. 2013, Article ID 247948, 16 pages, 2013.

[7] H. Hu, H.-Q. Tang, J.-H. Li, L.-L. Yang, W.-J. Tang, and Z.R. Zhou, "Efficacy and safety of Shen Song Yang Xin capsule for cardiac arrhythmia: a systematic review," Chinese Journal of Evidence-Based Medicine, vol. 11, no. 2, pp. 168-173, 2011.

[8] C.-H. Gu, Y.-L. Wu, S.-Y. Tian et al., "Effect of Shensong Yangxin capsule on ventricular premature beat and cardiovascular autonomic nervous function in patients with coronary heart disease," Chinese Journal of Integrated Traditional and Western Medicine, vol. 25, no. 9, pp. 783-786, 2005.

[9] S. B. Chai, S. R. Wang, L. F. Yao et al., "Effect of Shensong Yangxin capsule on ventricular remodeling in rats after myocardial infarction and isolated cardiac action potential," Bei Jing Journal of Traditional Chinese Medicine, vol. 28, no. 12, pp. 967970, 2009.

[10] N. Li, X. F. Wu, K. J. Ma et al., "The effect of Shensong Yangxin capsule on potassium channel currents in isolated ventricular myocytes," Chinese Journal of Difficult and Complicated Cases, vol. 6, no. 3, pp. 133-137, 2007.

[11] Z. Y. Jin, Q. Gong, J. L. Pu et al., "The effect of Shensong Yangxin capsule on the porcine cardiac electrophysiology," Chinese Patent Medicine, vol. 31, no. 3, pp. 471-473, 2009.

[12] J. P. T. Higgins and S. Green, Cochrane Handbook for Systematic Reviews of Interventions, Version 5.1.0, The Cochrane Collaboration, 2011, http://handbook.cochrane.org/.

[13] Y. F. Huang, "Clinical study of amiodarone combined with Shen Song Yang Xin capsule in treatment of elderly patients with frequent ventricular premature contractions in 64 cases," Sichuan Yi Xue Za Zhi l, vol. 30, no. 5, p. 724, 2009.

[14] R. Z. Guo, "The effect of amiodarone combined with Shen Song Yang Xin capsule in the treatment of frequent ventricular contractions," Journal of Practical Medical Techniques, vol. 15, no. 12, pp. 1552-1553, 2008. 
[15] R. S. Zhu, F. Xu, and Y. Fang, "Effect of Shen Song Yang Xin capsule as adjuvant treatment for patients with suspected sick sinus syndrome with frequent ventricular premature contractions," Chinese Journal of Difficult and Complicated Cases, vol. 11, no. 9, pp. 693-695, 2012.

[16] X. H. Ping, J. Zhou, and X. C. Zhang, "The effect of Shen Song Yang Xin capsule combined with amiodarone in the treatment of frequent ventricular contractions," Practical Clinical Medicine, vol. 12, no. 6, pp. 16-18, 2012.

[17] X. L. Su and R. Wang, "Clinical research of Shen Song Yang Xin capsule combined with mexiletine in treatment of patients with frequent ventricular premature contractions," Chinese Journal of Hospital Pharmacy, vol. 27, no. 3, pp. 369-370, 2007.

[18] J. Yu, Y. W. Yang, and Z. H. Ma, "Clinical research of Shen Song Yang Xin capsule combined with metoprolol in treatment of patients with frequent ventricular premature contractions," Chinese Journal of Practical Nervous Diseases, vol. 12, no. 13, pp. 96-98, 2009.

[19] L. P. Zhou and H. Li, "Effect of Shensong Yangxin capsule combined with metoprolol on ventricular arrhythmia," Chinese Journal of Ethnomedicine and Ethnopharmacy, vol. 23, no. 1, pp. 102-105, 2011.

[20] H. Geng, G. X. Zhao, and P. W. Zhao, "Clinical research of Shen Song Yang Xin capsule combined with metoprolol in treatment of elderly patients with frequent ventricular premature contractions," Chinese Journal of Clinical Rational Drug Use, vol. 4, no. 2A, pp. 65-66, 2011.

[21] D. M. Zhou, "Clinical research of Shen Song Yang Xin capsule combined with metoprolol for treatment of frequent ventricular premature contractions," Guide of China Medicine, vol. 11, no. 21, pp. 672-673, 2013.

[22] H. Wan, "Clinical effect of Shen Song Yang Xin capsule combined with metoprolol for treatment of frequent ventricular premature contractions," Traditional Chinese Medicine of Inner Mongolia, June 2012.

[23] L. R. Fu, M. W. Xia, and M. H. Xiao, "Clinical research of Shen Song Yang Xin capsule combined with small dose of amiodarone for treatment of frequent ventricular premature contractions," Journal of Modern Medicine and Health, vol. 26, no. 20, pp. 3090-3091, 2010.

[24] L. Qiu, X. S. Yang, and Q. Wang, “Clinical study of frequent premature ventricular contractions treated by Shenysong Yangxin capsule combined with low dose of metoprolol," Journal of Liaoning Traditional Chinese Medicine, vol. 39, no. 7, pp. 13451347, 2012.

[25] Y. H. Li and S. M. Yan, "Clinical study of Shen Song Yang Xin capsule combined with propafenone fot treatment of frequent ventricular premature contractions in 30 cases," China Pharmaceuticals, vol. 18, no. 13, p. 73, 2009.

[26] A. S. Fu, P. Lu, Y. C. Wang et al., "Clinical study of Shen Song Yang Xin capsule combined with amiodarone in the treatment of elderly frequent ventricular contractions," Clinical Misdiagnosis \& Mistherapy, vol. 23, no. 4, pp. 316-318, 2010.

[27] Y. Zhang, Q. Zhang, and H. P. Li, “Comparison observation on the therapeutic effects between Shensongyangxin capsule and betaloc on frequent premature ventricualr contraction," Sichuan Medical Journal, vol. 2, no. 8, pp. 260-262, 2011.

[28] M. H. Liu and C. A. Li, "Clinical study of Shen Song Yang Xin capsule for the treatment of frequent ventricular contractions," Seek Medical and Ask the Medicine, vol. 9, no. 6, p. 97, 2011.

[29] R. L. Nahin, P. M. Barnes, B. J. Stussman, and B. Bloom, "Costs of complementary and alternative medicine (CAM) and frequency of visits to CAM practitioners: United States, 2007,' National Health Statistics Reports, no. 18, pp. 1-14, 2009.

[30] S. M. K. Rates, "Plants as source of drugs," Toxicon, vol. 39, no. 5, pp. 603-613, 2001.

[31] X. J. Xiong, X. C. Yang, Y. M. Liu, Y. Zhang, P. Q. Wang, and J. Wang, "Chinese herbal formulas for treating hypertension in traditional Chinese medicine: perspective of modern science," Hypertension Research, vol. 36, pp. 570-579, 2013.

[32] J. Wang, P. Q. Wang, and X. J. Xiong, "Current situation and reunderstanding of syndrome and formula syndrome in Chinese medicine," Internal Medicine, vol. 2, no. 3, Article ID 1000113, pp. 1-5, 2012.

[33] J. Wang and X. J. Xiong, "Current situation and perspectives of clinical study in integrative medicine in China," Evidence-Based Complementary and Alternative Medicine, vol. 2012, Article ID 268542, 11 pages, 2012. 


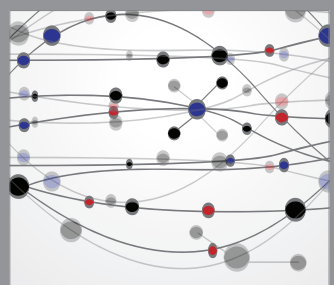

The Scientific World Journal
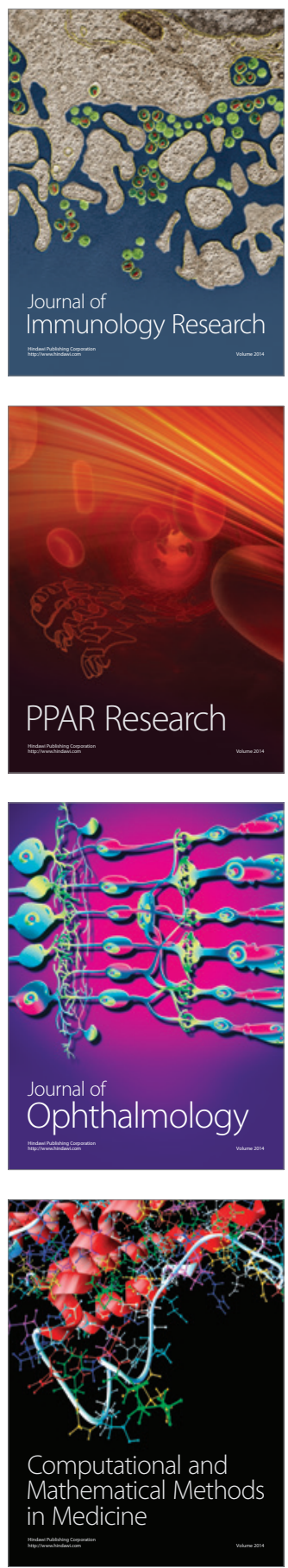

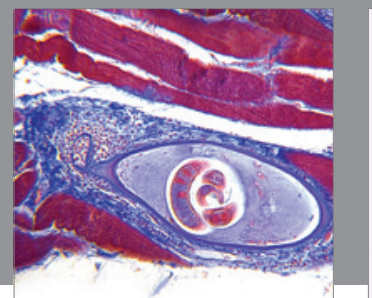

Gastroenterology

Research and Practice
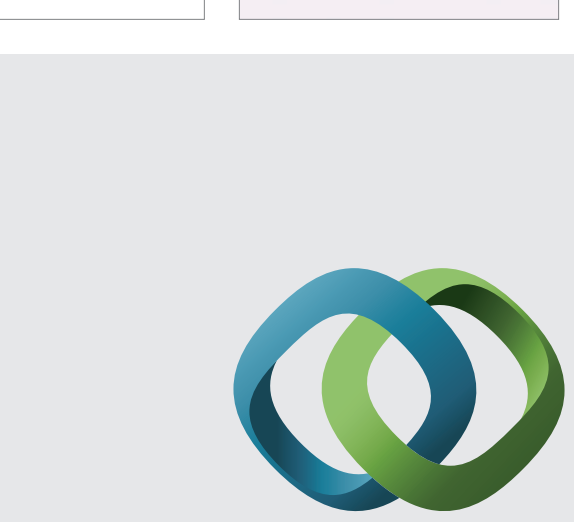

\section{Hindawi}

Submit your manuscripts at

http://www.hindawi.com
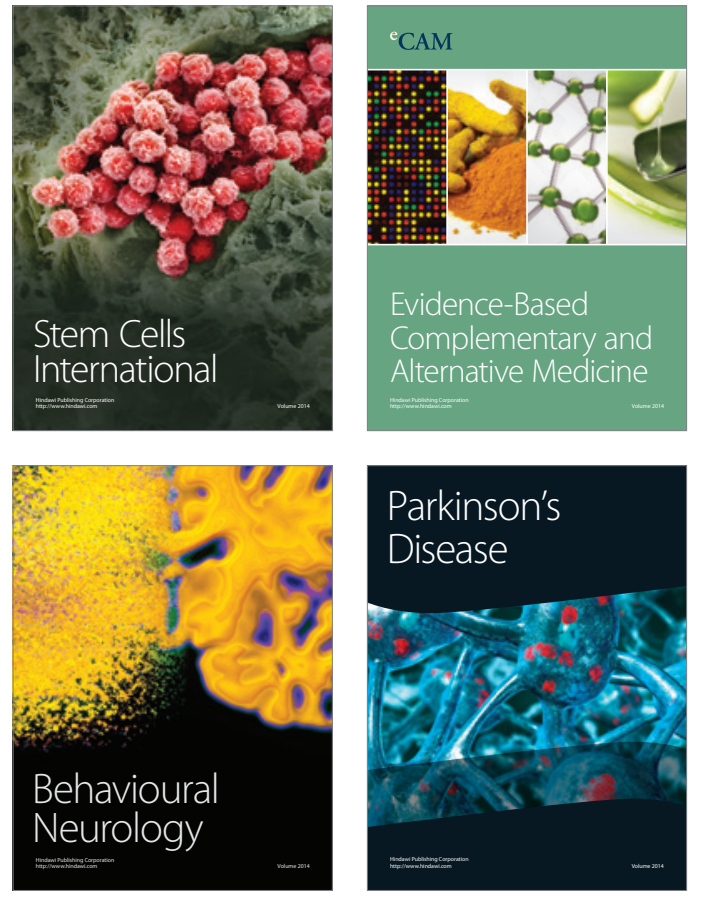
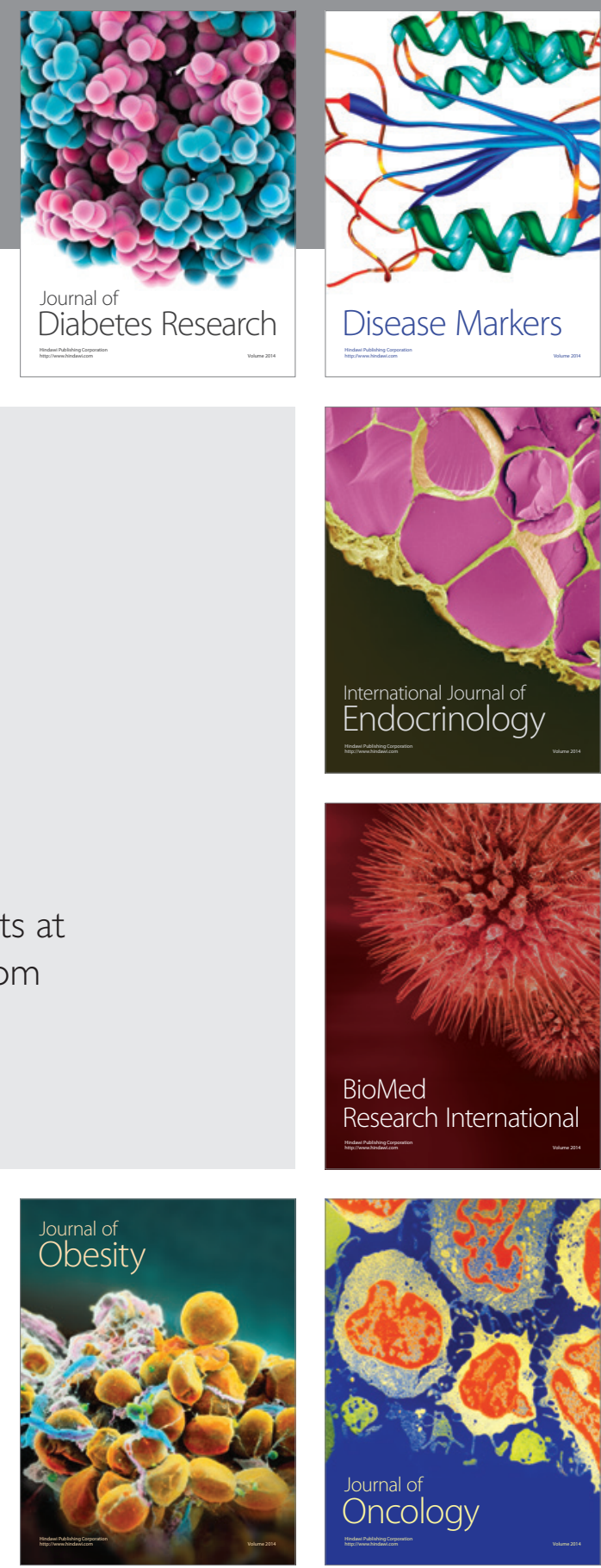

Disease Markers
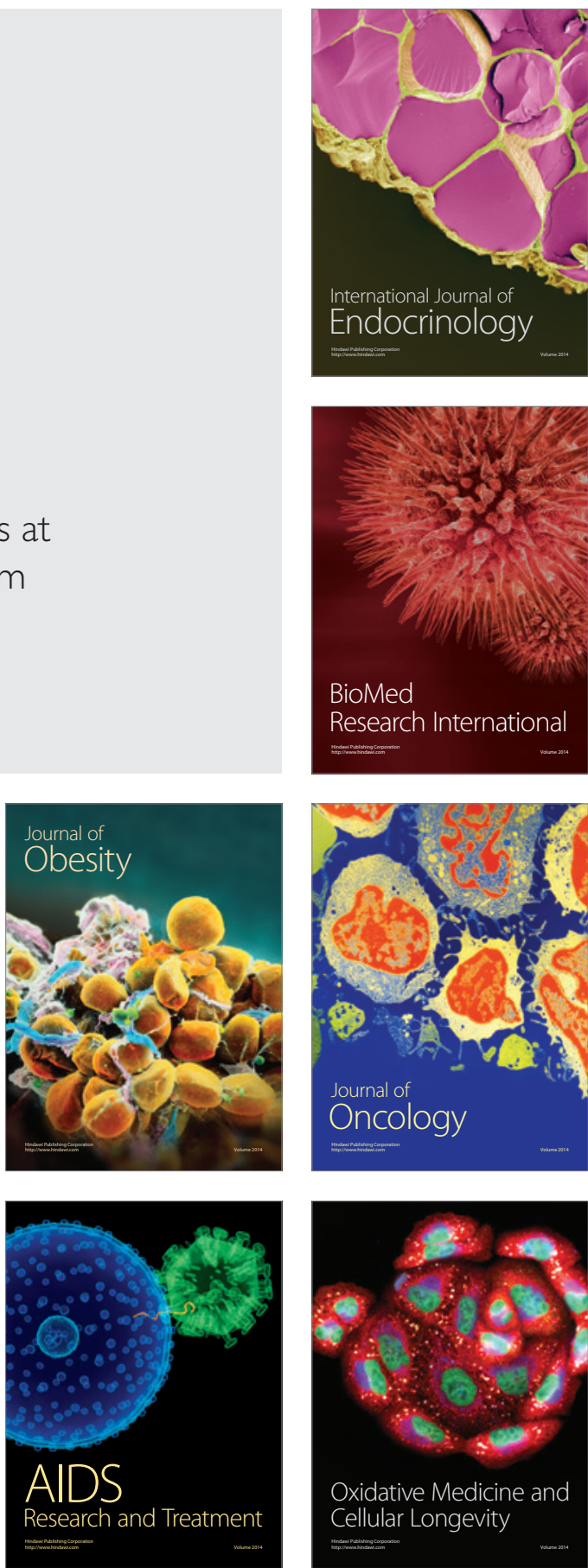